\title{
Surface Reconstruction of Wear in Carpets by Using a Wavelet Edge Detector
}

\author{
Sergio A. Orjuela Vargas ${ }^{\star 1,3}$, Benhur Ortiz Jaramillo ${ }^{4}$, Simon De \\ Meulemeester $^{2}$, Julio C. Garcia Alvarez ${ }^{4}$, Filip Rooms ${ }^{1}$, Aleksandra Pižurica ${ }^{1}$, \\ and Wilfried Philips ${ }^{1}$ \\ ${ }^{1}$ Department of Telecommunications and Information Processing , \\ (TELIN-IBBT), Ghent University, Belgium, \\ ${ }^{2}$ Department of Textiles, Ghent University, Belgium \\ \{SergioAlejandro.OrjuelaVargas, Simon.DeMeulemeester, Filip. Rooms, , \\ Aleksandra.Pizurica, Wilfried.Philips\}@UGent.be, \\ ${ }^{3}$ Perception and Robotics Group, Antonio Nariño University, Colombia \\ ${ }^{4}$ Control and Digital Signal Processing Group, National University, Colombia \\ \{bortizj, jcgarciaa\}@unal.edu.co
}

\begin{abstract}
Carpet manufacturers have wear labels assigned to their products by human experts who evaluate carpet samples subjected to accelerated wear in a test device. There is considerable industrial and academic interest in going from human to automated evaluation, which should be less cumbersome and more objective. In this paper, we present image analysis research on videos of carpet surfaces scanned with a 3D laser. The purpose is obtaining good depth images for an automated system that should have a high percentage of correct assessments for a wide variety of carpets. The innovation is the use of a wavelet edge detector to obtain a more continuously defined surface shape. The evaluation is based on how well the algorithms allow a good linear ranking and a good discriminance of consecutive wear labels. The results show an improved linear ranking for most carpet types, for two carpet types the results are quite significant.
\end{abstract}

Keywords: Carpet wear, LBP technique, Kullback-Leibler, Image analysis, Automated labeling assessment

\section{Introduction}

One of the most important parameters for the classification of the quality of floor coverings is the conservation of appearance after an accelerated wear process. To assess their aspect preservation, textile floor coverings were initially compared with previously rated reference samples. As the physical features of

\footnotetext{
* Sergio Orjuela is supported by a grant of the LASPAU Academic and Professional Programs for the Americas in agreement with the COLCIENCIAS Science \& Technology Program, Colombia.
} 
these reference samples may change over time, a normalization committee decided to propose certified photographs by international committees instead. In the assessment process, the carpets are first subjected to accelerated mechanical wear to simulate traffic exposure. Consequently, a group of trained experts compare the original carpet with the carpet subjected to an accelerated mechanical wear. Experts evaluate the wear level from color appearance and 3D structure, attributing values between 1 and 5 to each carpet. Value 1 corresponds to a total loss of structure and value 5 is attributed to a carpet that did not undergo a structure change. This visual evaluation method lacks in reproducibility and the method requires at least three experts, which is not always possible, especially within a small company. The human evaluation assessment is also somewhat subjective and industry is very interested in converting these traditional standards to automated objective standards. However, no automated system exists yet to enable the labeling process.

Research in automated rating has progressed, specifically on image analysis for extracting texture features from digital color images. Approximations to the human evaluation have been achieved using Fourier transform [1], second order statistics [2], random markov fields [3] and neural networks [4]. However, practical applications were limited at that time by factors such as the amount of incorrect assessments exceeding $5 \%$ and algorithms being considered not generic enough for a broad variety of carpets.

Recently, some researchers have been exploring the use of depth information capturing the three dimensional structures of the carpets also evaluated by the experts. Initially, a scanner for 3D objects was used to capture the 3D shape of the carpet surfaces [5]. However, with that scanner the depth data were captured into nonstructured grids, with the number of acquired points highly dependent on the colors of the object. To extract texture features from these data using image analysis, the information of depth had to be structured first into $2 \mathrm{D}$ images. This has two disadvantages, an additional computational cost and the possibility of distortion of the surface shape due to interpolation methods involved in this process [6]. Although a classification over 95\% was achieved, the extracted features did not change accordingly with the wear labels as should be expected. Nevertheless, research in automating the labeling process now attempts to find a universal classification system for carpets using depth and/or intensity data.

To optimize the feature extraction process a methodology based on experimental design has been proposed [7]. The methodology assumes that for a correct description the features must not only change monotonically according to the wear labels but also must be highly discriminate between consecutive wear labels.

This methodology has been tested extracting Local Binary Pattern statistics on references from different standards $[8,9]$, obtaining rank correlations over 0.9 , linear correlations over 0.88 and a $89 \%$ of statistical distinction between consecutive wear labels. Additionally, a novel 3D scanner based on structured light for specifically scanning carpets has been developed [10]. 3D data, termed range data, are obtained by video capturing with a camera the reflection of a uniform laser line on the surface of the carpet. Then, the depth information is 
calculated from the highest position of the brightest reflection on the columns of each frame. This system offers better linear and linear-rank correlations between wear labels and texture features computed from depth data. Experiments suggest that depth and color information are complementary to generalize the description on several carpets [11]. However, discrimination is still better using data from intensity images.

In this paper we present our proposed method to improve the surface representation on depth images. The method increases the discriminance of texture by extracting the depth from the $3 \mathrm{D}$ range data using edge detection based on multiresolution analysis. Thus, the relevant edges can be detected and separated from noise. This type of analysis offers excellent results for texture analysis and classification [12-16] and will be shown that the proposed method achieves a better linear ranking of wear labels than the previous method for all carpet types. We compare the results of our proposed method to the method applied in [10] as well as the optimal results from both methods to results using images of intensity.

The paper is organized as follows. In Section 2 we describe the materials used in this apporach. In Section 3 we describe the method that we propose to improve the construction of depth images as well as the methodology employed to compare the methods. In Section 4 we report the results of the comparison. Finally, in Sections 5 findings are discussed and conclusions are drawn.

\section{Materials}

In this approach we use eight types of reference sets provided from the EN1471 standard. Each reference contains a collection of samples of a particular textile floor covering priorly subjected to different revolutions on the Vettermann tester. The samples have been collected from carpet types loop, cut/loop, woven cut pile, fris and shaggy. Each reference includes eight labelled samples of transitional degrees of wear. These sets do not include label 5 and the labels vary from 4.5 to 1 , with steps of half point. Label 4.5 represents a minor change in appearance and label 1 a several change.

We composed a database of videos using the scanner based on structured light. For this, the samples were scanned one sample at a time. The sample was first vacuum cleaned and held with elastic bands upon an inox-drum with a diameter of $25 \mathrm{~cm}$. On the surface of the sample a uniform line was projected. The line was produced with a line laser generator model LDM-4-650-1-PF200UL60. This type of lasers produce a bright, crisp laser line of uniform intensity with a fan angle of $60^{\circ}$. It was fixed at $20 \mathrm{~cm}$ above the drum projecting on the surface of the sample a high quality uniform line with a length of $20 \mathrm{~cm}$ and with a thickness of $0.5 \mathrm{~mm}$.

The reflection of the line was seen with a progressive 3CCD Sony camera model DXC-9100P using a Sony macro lens model VCL-707BXM. The camera was fixed at a distance of $30 \mathrm{~cm}$ from the line to the objective lens. Figure 1 shows the reflection of the uniform laser line on the surface of samples from 
the same reference with different degrees of change in appearance. To cover the surface of the sample with the line, the drum was moved using a motor model Parvalux 400-753 with gear box. This allowed us to control the distance between captured lines. The speed of the motor was controlled using an AC Inverter Drive Speed Controller with model Parker SSD Drives 650. Thus, 50 seconds of video have been recorded for each sample with $25 \mathrm{fps}$. The frames are spaced $0.24 \mathrm{~mm}$ on the surface, each frame with a resolution of $720 \times 576$ pixels.

As a result we have composed a database of 64 videos, capturing the $3 \mathrm{D}$ structure of eight types of reference fatigued specimens. Each reference with samples of eight wear labels. Figure 1 displays sections of frames showing the change of appearance on the surface of reference samples.

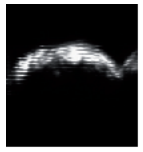

4.5

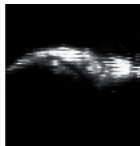

4.0

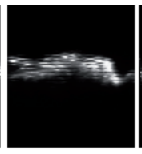

3.5

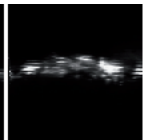

3.0

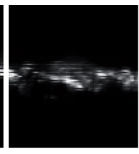

2.5

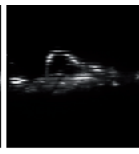

2.0

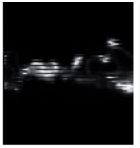

1.5

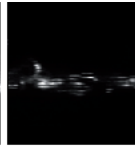

1.0

Wear Labels

Fig. 1. Surface appearance change captured by the camera on carpet type loop.

\section{Methods}

To be able to use classic image analysis, the depth information given by the reflection of the light must be stored into an image. For this, Orjuela et al. have proposed in [10] and [11] to search in each column of the frame for the highest position of the highest intensity value corresponding to the reflected light on the surface. That position represents the depth and an array is constructed using one depth value per column from the frame. Then, the image is built adding the arrays when the drum is rotating. In the following, we will refer to this method as the RefLight method.

From Figure 1 it can be observed that the reflected light forms discontinuous segments with a high variation in width. We are however particularly interested in obtaining fine lines representing small details that describe changes in surface between wear labels. Using the RefLight method on this kind of images has two disadvantages. The first one is that it is highly dependent on the intensity of the reflected light; the second one is that the detected points are unconnected between columns since they are independently calculated. Since this method does not assure a good reconstruction with a continuous surface shape, we propose to use an edge detection procedure instead.

For a better reconstruction of the surface shape, we propose to detect the edge of the laser line frame by frame using a wavelet representation [17]. The 
need for a multiresolution evaluation relies on the fact that edges such as those describing the reflection of the light are optimally detected by using different scales and classical edge detectors (like Canny) only use a single scale parameter for the whole image [18]. Thus, the basic single scale edge detection has been extended to an approach that combines information about the behaviour of the edges across a set of different scales [19]. The number of scales is determined by applying a Gaussian lowpass filter with increasing standard deviation, termed $\sigma$, from 1 to 5 until separate fibers can no longer be distinguished in the image [20]. In our case the separated fibers are no longer perceptible beyond $\sigma=3$. This is illustrated in Figure 2.

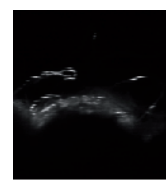

Original

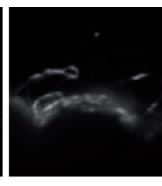

$\sigma=1$

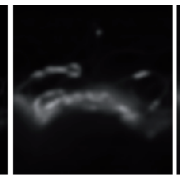

$\sigma=2$

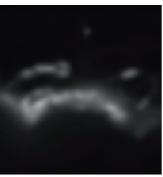

$\sigma=3$

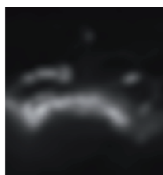

$\sigma=4 \quad \sigma=5$

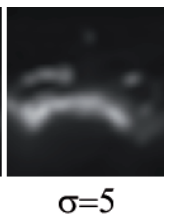

Fig. 2. Visual selection of the number of scale to evaluate.

The edges are detected in each sub-band by using a Canny second-order filter. Additionally, lower resolution sub-bands are merged by using a Sobel filter in order to preserve the position of the edge in the image. This filter is used because the edge is selected when the amplitude of the filter response increases from the finer to the lower scale sub-bands and eliminated when the amplitude decreases from finer to coarser scales [17]. In a more formal way, we compute the Lipschitz exponents for all positions in the image. This Lipschitz exponent expresses the local regularity of an image at a certain location. The Lipschitz exponent is small where only fine texture or noise are present and large where smoother features or continued edges are present. Recent works that use the Lipschitz exponent in multiresolution edge detection can be found in [21] and [22]. Using the Lipschitz exponent implicitly separates the noise from the edges. In the following, we will refer to this method for detecting edges as the EdgeWav method. Comparative methods can be found in [20,23], where merging is stated with an additional training phase in order to select the edges. For this application, only the moduli of edge pixel coefficients are required.

For the methods considered in this paper, depth images are again constructed by adding arrays when the drum is rotating, as it is defined in [10] and [11]. Thus, in the EdgeWav method, the highest position of the edges instead of the highest intensity value is searched in each column of the frame. This improves the representation of the depth of the surface by using connected points between adjacent columns. Figure 3 shows cut out examples of the change in appearance of the surface captured with both methods. 

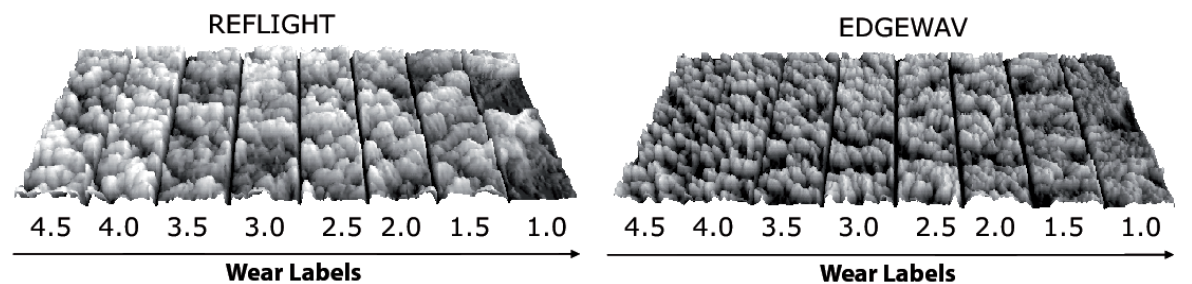

Fig. 3. Comparison of the reconstruction of the surface on wear labels of carpet type shaggy.

We want to compare the performance of both methods EdgeWav and RefLight in discriminating between consecutive wear labels and in correctly ranking them. For this, the texture transitions due to wear are first numerically represented by extracting texture features from the images. Then, we use two measures that quantify characteristics from the description of both methods. Finally, we evaluate which method offers the closest description of the wear label to the visual analysis by comparing both the measures.

To extract texture features, we compute a symmetrized adaptation of the Kullback-Leibler (SKL) [24] divergence on the distribution of Local Binary Patterns (LBP) [7] from the images. We use the Rotational, Mirrored and Complemented invariance extension of the LBP technique (LBPRMC) [7]. This technique has been tested on both intensity and depth images, revealing good performance for multiple types of carpets $[7,11]$. To characterize the texture, relationships between points on a circle around a central pixel are evaluated. Equally spaced points are considered on the circle and a corresponding pixel value in each point is interpolated from the four pixels closest to the point. Each point gets a code bit 0 or 1 assigned depending on whether it has a higher or lower gray value than the central pixel. These bits are read out clock wise and placed in a diadic code word named pattern. Mirror and complement versions of patterns are grouped using a look up table. This is because a carpet reflected in a mirror or with the colors inverted would be assigned the same rating by human assessors. The distribution of the patterns corresponding to wear labels are represented with histograms. In human visual inspection samples with changes in appearance are compared to samples of original appearance. However, there are not samples from original appearance in the database. We estimate the LBPRMC histograms corresponding to original appearance by using the pattern distributions of the respective changes in appearance, with wear labels from 1.0 to 4.5 . Thus, the bin values for the histogram corresponding to label 5.0 are estimated using a linear regression model since the frequency of a pattern is expected to change monotonically from labels 1.0 to 5.0 [7]. We quantify the difference in texture between the original appearance and the changes by comparing the corresponding LBPRMC histograms. We compute the symmetric Kullback-Leibler difference, termed $\kappa$, between two histograms as follows: 


$$
\kappa=\sum_{i=1}^{N} h_{1}(i) \log h_{1}(i)+\sum_{i=1}^{N} h_{2}(i) \log h_{2}(i)-\sum_{i=1}^{N} h_{p}(i) \log h_{p}(i)
$$

where $h_{1}(i)$ and $h_{2}(i)$ are the two histograms, which must be normalized first, $i$ is the bin index, $h_{p}(i)=h_{1}(i)+h_{2}(i)$ and $N$ is the total number of bins.

For each image in the database, we composed a set of 32 image variations, consisting in combinations of complements, rotations of $0,90,180,270$ degrees and mirrored up-down and left-right. Some of these $\kappa$ values from the same worn sample can be significantly larger or smaller than the others because of the presence of factors such as flecks or speckles that could not be removed by the vacuum cleaning. These outliers are detected using the Mahalanobis distance and then replaced with the associated median to allow statistical comparisons with an equal number of $\kappa$ values per worn sample.

To be consistent with the visual analysis, the $\kappa$ values are expected to change monotonically with the wear labels as well as to be clearly distinguished between consecutive wear labels [7]. To compare the WavEdge to the Reflight Method, we quantify both characteristics from the description of both methods.

The first characteristic is satisfied if the relation between $\kappa$ values and the wear labels is at least linear-ranked. To check this, we compute the Spearman linear-rank correlation between the wear labels and the mean values of the associated $\kappa$ values. The Spearman rank correlation assign a significance of correspondence between -1 to 1 , with 1 indicating a perfect rank correlation.

The second characteristic can be evaluated by checking the number of Consecutive Wear labels that can be Statistically Discriminated, termed CWSD [7]. This number can be quantified by counting how many times the difference between consecutive means is larger than the threshold for a statistic significance. The statistic significance is computed based on the Tukey test which allows pairwise comparisons. For this, an equal number of $\kappa$ values per wear label is required.

Most of the changes due to wear appear in the tips of the pile yarns tufts. As their size depends of the type of carpet, a multiresolution analysis is required [7]. This can be achieved by changing the number of circular neighbors and their distance from the center pixel [25]. However, we prefer then to keep these parameters fixed and to resize the images instead. To resize, we use bilinear interpolation, analysing pictures on scale factors from 0.6 to 1 with intervals of 0.1 . We present results only in this range because experiments show that the optimal resolution is found to always lie in it.

The linear rank correlation and CWSD are called the response variables and are computed for each one of the combinations of the elements shown in Table 1.

We are interested in evaluate whether the EdgeWav method offers a more monotonic and higher discriminant representation of the $\kappa$ values related to wear labels than the RefLight method. For this, we independently evaluate the response variables using ANalysis Of VAriance tests (ANOVA) [26]. We test for each response variable the null hypothesis ' $\kappa$ values related to wear labels offer the same representation for both methods'. ANOVA results are probabilities, 
Table 1. Elements evaluated in this experiment.

\begin{tabular}{|c|c|c|}
\hline $\begin{array}{c}\text { Method } \\
\text { Type }\end{array}$ & $\begin{array}{c}\text { Scale } \\
\text { Factor }\end{array}$ & $\begin{array}{c}\text { Reference } \\
\text { Type }\end{array}$ \\
\cline { 3 - 3 } RefLight & 0.6 & Shaggy1 \\
EdgeWav & 0.7 & Cut/loop \\
& 0.8 & Shaggy2 \\
0.9 & High/Low loop \\
1.0 & Frisé \\
& & Cut \\
& Loop \\
& Cut Design \\
\hline
\end{tabular}

termed $p$-values, where a $p$-value less than a given $\alpha$ rejects the null hypothesis, meaning that there is a significant difference in the response variables of both methods with $100(1-\alpha) \%$ of confidence.

For valid $p$-values, the methods must be independent of scale factors and carpet types. These dependences are identified also by ANOVA evaluating the null hypothesis 'there are no dependences between combinations of methods in Table 1', with $p$-values, where a $p$-value less than a given $\alpha$ rejects the null hypothesis, meaning that there are dependences between the associated elements. In case of dependences, the group is subdivided performing analysis in each subgroup that does not reveal dependences among methods and scale factors and/or carpet types. This assures the validity of the $p$-values.

\section{Results}

The ANOVA results show that using the EdgeWav method to construct depth images improves significantly the discriminance between consecutive wear labels as well as the linear rank between $\kappa$ values and wear labels. The results also show that the method works well for seven of the eight carpet types evaluated.

To obtain these results, we tested for significant differences in the response variables computed from images constructed with both the RefLight and the EdgeWav method. Therefore, we first checked for dependencies between the type of construction method and the type of carpets. During this analysis, the scale factors were not evaluated since there was no evidence that scale factors are dependent on either type of carpet or type of method. Afterwards, we searched in each type of carpet for the best scale factor using only images constructed with the EdgeWav method. In the following, we will describe the ANOVA results in detail.

- Evaluation of Linear Rank Correlation (LRC). The ANOVA test shows dependences between the type of methods and the type of carpets ( $p$-value $<0.01)$. However, there is no evidence of dependences between scale factors and the other two elements $(p$-value $=0.894$ for dependences with methods and $p$-value $=0.252$ for dependences with type of carpets ). There is also no evidence at this point of significant differences between scale factors $(p$-value $=0.617)$. From this, we separated the type of carpets in the following three groups: 
1. Carpets with high significant differences in LRC. This group is composed of carpets in which the EdgeWav method improves over 0.1 the linear rank correlation. Carpet type shaggy1 and cut/loop are in this group. There is no evidence in this group of dependences between carpet types and methods $(p$-value $=0.24>0.5)$ and the LRC values are identified to be significantly different $(p$-value $>0.01)$.

2. Carpets with low significant differences in LRC. This group is composed of carpets in which the EdgeWav method improves less than 0.1 the linear rank correlation. There is no evidence in this group of dependences between carpet types and methods $(p$-value $=0.067>0.5)$ and the LRC values are identified to be significantly different $(p$-value $>0.01)$. This group contains carpet types shaggy2, high/low loop and frisé.

3. Carpets with no significant differences. This group is composed of carpets in which the EdgeWav method does not improve the linear rank correlation. There is no evidence in this group of dependences between carpet types and methods $(p$-value $=0.89>0.5)$. There is neither evidence in this group of significant differences in LRC values $(p$-value $=$ $0.89>0.5)$. This group is composed of carpet types loop and cut design

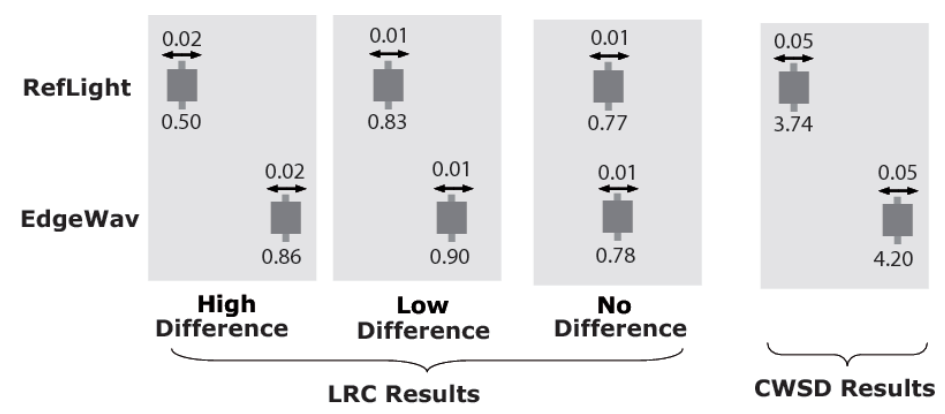

Fig. 4. Comparison of linear rank correlations between the RefLight and the EdgeWav methods.

- Evaluation of the number of Consecutive Wear labels that can be Statistically Discriminated (CWSD). The ANOVA results shows no evidence of dependences between carpet types and methods ( $p$-value $=$ $0.091>0.5)$. CWSD values are identified to be significantly different ( $p$-value $>0.01$ ) for all the eight types of carpets.

Figure 4 summarize the ANOVA results. Mean values, at the bottom of the boxes, and standard deviations, represented with $\leftrightarrow$ at the top of the boxes, are shown.

- Selecting for each type of carpet the optimal scale factor. As a final step we choose the scale factor with the best combination of response variables. Thus, the wear labels of the eight types of carpets are optimally 
represented with the combination presented in Table 2 . The table lists the quantified characteristics, including linear correlations values for comparison purposes with other approaches. Results obtained in [11] are also displayed to state the advantages of the EdgeWav method over the RefLight method as well as to compare the wear label description from depth images constructed using the EdgeWav method to the wear label description obtained using intensity images. The values corresponding to the EdgeWav method proposed in this approach are highlighted in italics. Additionally, for cases where EdgeWav performs better than intensity images, the values have been highlighted in bold.

Table 2. Optimal representation chosen for the eight types of carpets. LRC specifies how well the $\kappa$ values are ranked according to the wear labels, CWSD determinates the number of $\kappa$ related to consecutive wear labels that can be statistically discriminated and LC is the linear correlation between $\kappa$ values and wear labels.

\begin{tabular}{|c|c|c|c|c|c|}
\hline $\begin{array}{c}\text { Carpet } \\
\text { Type }\end{array}$ & $\begin{array}{c}\text { Image } \\
\text { Type }\end{array}$ & $\begin{array}{c}\text { Scale } \\
\text { Factor }\end{array}$ & LRC & CWSD & LC \\
\hline \multirow{3}{*}{ Shaggy1 } & Edge Wav & $\boldsymbol{0 . 7}$ & $\mathbf{0 . 9 7}$ & $\mathbf{5 . 0 0}$ & $\mathbf{0 . 9 3}$ \\
& RefLight & 0.7 & 0.93 & 4.00 & 0.92 \\
& Intensity & 0.7 & 0.93 & 4.60 & 0.75 \\
\hline \multirow{3}{*}{ Cut/loop } & Edge Wav & $\mathbf{0 . 6}$ & $\mathbf{0 . 9 6}$ & $\mathbf{5 . 0 0}$ & $\mathbf{0 . 9 6}$ \\
& RefLight & 0.7 & 0.53 & 4.00 & 0.48 \\
& Intensity & 0.9 & 0.49 & 5.20 & 0.49 \\
\hline \multirow{3}{*}{ Shaggy2 } & EdgeWav & 0.9 & 0.87 & 4.40 & 0.89 \\
& RefLight & 0.9 & 0.77 & 4.00 & 0.80 \\
& Intensity & 1.0 & 0.92 & 5.60 & 0.87 \\
\hline \multirow{3}{*}{ Frisé } & EdgeWav & 0.6 & 0.95 & 4.20 & 0.96 \\
& RefLight & 0.6 & 0.88 & 4.00 & 0.93 \\
& Intensity & 0.9 & 0.99 & 6.40 & 0.97 \\
\hline \multirow{3}{*}{ Cut } & EdgeWav & $\mathbf{0 . 9}$ & $\mathbf{0 . 9 9}$ & $\mathbf{6 . 2}$ & $\mathbf{0 . 9 9}$ \\
& RefLight & 0.9 & 0.97 & 5.40 & 0.97 \\
& Intensity & 1.0 & 0.89 & 5.20 & 0.82 \\
\hline \multirow{2}{*}{ Loop } & EdgeWav & 0.8 & 0.99 & 5.00 & 0.91 \\
& RefLight & 0.9 & 0.95 & 3.60 & 0.93 \\
& Intensity & 1.0 & 0.99 & 6.00 & 0.96 \\
\hline \multirow{2}{*}{ Cut Design } & Edge Wav & $\mathbf{1 . 0}$ & $\mathbf{1 . 0 0}$ & $\mathbf{6 . 6 0}$ & $\mathbf{0 . 9 1}$ \\
& RefLight & 0.8 & 1.00 & 6.60 & 0.94 \\
& Intensity & 0.9 & 0.98 & 6.20 & 0.97 \\
\hline & EdgeWav & 0.9 & 0.70 & 4.00 & 0.68 \\
& RefLight & 1.0 & 0.65 & 3.80 & 0.73 \\
& Intensity & 0.9 & 0.87 & 4.20 & 0.81 \\
\hline
\end{tabular}

\section{Discussions and conclusions}

In this paper a comparison between two methods for reconstructing the surface of carpets from depth images has been presented. The novel use of a wavelet edge detection algorithm has been compared to a previously used algorithm that only looked at the highest position of the brightest reflection in the images. The images were taken from samples of 8 types of carpets provided from the EN1471 standard. The texture features were extracted comparing the distribution of LBP 
patterns between images of original and change in appearance of carpets. Then two characteristics were used to evaluate the algorithms against each other, these characteristics are first how well the relation of the $\kappa$ values (obtained from the Symmetric Kullback-Leibler difference) and the wear labels is linear ranked and second how well consecutive wear labels can be statistically discriminated using their $\kappa$ values. The results of this evaluation show that the wavelet edge detector significantly improves the linear ranking compared to the original algorithm for six of the eight carpet types. For two carpet the method tend to perform better but without statistical evidence of significant improvement. The statistical discriminance between consecutive wear labels improves for all types of carpets. As a last step, the optimum (giving best response variables) scale factor of the images was researched and both characteristics and linear correlation coefficient were given. These measures permit comparison of results from images obtained with our method to results using depth images obtained with the original method as well as intensity intensity images, both evaluated in [11]. Wear labels in four carpet types are best represented with depth images and the other four with intensity images. This confirms that the texture features from both types of images are complementary for representing the wear label. Particularly, wear labels in carpet type cut/lop could be now represented, which was not possible in the previous approach. We have shown that using the wavelet edge detector, texture features with a more monotonical description with wear labels can be obtained for depth images. These types of features combined with features from intensity images can be used for developing an automated carpet assessment system dealing with multiples carpets.

\section{References}

1. J. Wang and E.J. Wood. A new method for measuring carpet texture change. Textile Research Journal, 65:196-202, April 1994.

2. J. Sobus, B. Pourdeyhimi, J. Gerde, and Ulcay Y. Assessing changes in texture periodicity due to appearance loss in carpets: Gray level co-occurrence analysis. Textile Research Journal, 61:557-567, October 1991.

3. J. Wang, R. A. Campbell, and R. J. Harwood. Automated inspection of carpets. In Proc. SPIE, Optics in Agriculture, Forestry, and Biological Processing, volume 2345, pages 180-191. SPIE, January 1995.

4. S. Sette, L. Boullart, and P. Kiekens. Self-organizing neural nets: A new approach to quality in textiles. Textile Research Journal, 65:196-202, April 1995.

5. W. Waegeman, J. Cottyn, B. Wyns, L. Boullart, B. De Baets, L. Van Langenhove, and Detand J. Classifying carpets based on laser scanner data. Engineering Applications of Artificial Intelligence, 21(6):907-918, September 2008.

6. C. Copot, S. Syafiie, S. A. Orjuela, R. De Keyser, Van Langenhove L., and C. Lazar. Carpet wear classification based on support vector machine pattern recognition approach. In IEEE Int. conference on Intelligent Computer Communication and Processing, 5th, Proceedings, pages 161-164, 2009.

7. S. A. Orjuela, E. Vansteenkiste, F. Rooms, S. De Meulemeester, R. De Keyser, and W. Philips. Evaluation of the wear label description in carpets by using local binary pattern techniques. Textile Research Journal, published online, 2010. 
8. The Carpet and Rug Institute. Assessment of carpet surface appearance change using the cri reference scales. Technical Bulletin, 2003.

9. European Committee for standardization. Constructional details of types of textile floor covering available as reference fatigued specimens, 1996.

10. S. A. Orjuela, E. Vansteenkiste, F. Rooms, S. De Meulemeester, R. De Keyser, and W. Philips. Feature extraction of the wear label of carpets by using a novel 3d scanner. In Proc. of the Optics, Photonics and Digital Technologies for Multimedia Applications conference, 2010.

11. S. A. Orjuela, E. Vansteenkiste, F. Rooms, S. De Meulemeester, R. De Keyser, and W. Philips. A comparison between intensity and depth images for extracting features related to wear labels in carpets. In Proc. of SPIE Applications of Digital Image Processing XXXIII, volume 7798, 2010.

12. J. Luo and A. Savakis. Texture-based segmentation of natural images using multiresolution autoregressive models. In IEEE Western New York Image Processing Workshop, September 1998.

13. K. Daoudi, A. B. Frakt, and A. S. Willsky. An efficient method for texture defect detection: sub-band domain co-occurrence matrices. Image and Vision Computing, 18(6-7):543-553, May 2000.

14. Y. Han and P. Shi. An adaptive level-selecting wavelet transform for texture defect detection. Image and Vision Computing, 25(8):12391248, July 2007.

15. Z.-Z. Wang and J.-H. Yong. Texture analysis and classification with linear regression model based on wavelet transform. IEEE Trans. Image Processing, 17(8):14211430, August 2008.

16. Z. He, X. You, and Y. Yuan. Texture image retrieval based on non-tensor product wavelet filter banks. Signal Processing, 89(8):15011510, August 2009.

17. S. Mallat and W. L. Hwang. Singularity detection and processing with wavelets. IEEE Trans. Information Theory, 38(2):617-643, March 1992.

18. J. H. Elder and S. W. Zucker. Local scale control for edge detection and blur estimation. IEEE Trans. Pattern Analysis and Machine Intelligence, 20(7):699716, July 1998.

19. J. Li. A wavelet approach to edge detection. Master's thesis, Sam Houston State University, August 2003.

20. C. Ducottet, T. Fournel, and B. Barat. Scale-adaptive detection and local characterization of edges based on wavelet transform. Signal Processing, 84(11):21152137, November 2004.

21. P. Bao, L. Zhang, and X. Wu. Canny edge detection enhancement by scale multiplication. IEEE Trans. on Pattern Analysis and Machine Intelligence, 27(9):14851490, September 2005.

22. L. Xue and J. Pan. Edge detection combining wavelet transform and canny operator based on fusion rules. In Proc. of Wavelet Analysis and Pattern Recognition, 2009.

23. O. Laligant, F. Truchetet, J. Miteran, and P. Gorria. Merging system for multiscale edge detection. Optical Engineering, 44(3):035602-1,035602-11, March 2005.

24. M. Petrou and P. G. Sevilla. Image Processing Dealing with Texture. Wiley, January 2006.

25. T. Ojala, M. Pietikäinen, and T. Mäenpää. Multiresolution gray scale and rotation invariant texture classification with local binary patterns. IEEE Trans. on Pattern Analysis and Machine Intelligence, 24(7):971-987, July 2002.

26. M. Kutner, C. J. Nachtsheim, J. Neter, and Li W. Applied Linear Statistical Models. McGraw-Hill/Irwin, 5 edition, 2004. 